\title{
Quantitative study of optical pumping in the presence of spin-exchange relaxation
}

\author{
Yongqi Shi, ${ }^{1,2,3, *}$ Theo Scholtes, ${ }^{1}$ Zoran D. Grujić, ${ }^{1}$ Victor Lebedev, ${ }^{1}$ Vladimir Dolgovskiy, ${ }^{1}$ and Antoine Weis ${ }^{1, \dagger}$ \\ ${ }^{1}$ Physics Department, University of Fribourg, Chemin du Musée 3, CH-1700 Fribourg, Switzerland \\ ${ }^{2}$ University of Chinese Academy of Sciences, Beijing 100049, China \\ ${ }^{3}$ Institute of Electronics, Chinese Academy of Sciences, Beijing 100190, China
}

(Received 22 November 2017; published 24 January 2018)

\begin{abstract}
We have performed quantitative measurements of the variation of the on-resonance absorption coefficients $\kappa_{0}$ of the four hyperfine components of the Cs $D_{1}$ transition as a function of laser power $P$, for pumping with linearly and with circularly polarized light. Sublevel populations derived from rate equations assuming isotropic population relaxation (at a rate $\gamma_{1}$ ) yield algebraic $\kappa_{0}(P)$ dependences that do not reproduce the experimental findings from Cs vapor in a paraffin-coated cell. However, numerical results that consider spin-exchange relaxation (at a rate $\gamma_{\mathrm{se}}$ ) and isotropic relaxation fit the experimental data perfectly well. The fit parameters, viz., the absolute value of $\kappa_{0}$, the optical pumping saturation power $P_{\text {sat }}$, and the ratio $\gamma_{\mathrm{se}} / \gamma_{1}$, are well described by the experimental conditions and yield absolute values for $\gamma_{1}$ and $\gamma_{\mathrm{se}}$. The latter is consistent with the previously published Cs-Cs spin-exchange relaxation cross section.
\end{abstract}

DOI: 10.1103/PhysRevA.97.013419

\section{INTRODUCTION}

Optical pumping, invented in the 1950s and honored by the 1966 Nobel Prize in physics [1], is a method by which the rotation and/or reflection symmetry of a light beam that is resonant with an atomic medium is transferred onto the medium by subsequent photon absorption and/or reemission cycles. When pumped with circularly polarized light, the medium will acquire in this way a vector spin polarization (orientation), which has the axial symmetry of the light polarization. When pumped with linearly polarized light, the medium acquires a second-rank tensor polarization (alignment), with rotational invariance around the polarization axis and reflection invariance with respect to a plane perpendicular to it.

Since atomic magnetic moments are parallel or antiparallel to the atomic spin polarization, optical pumping is an efficient method for magnetizing a dilute (vapor, beam, or even liquid) atomic sample. The interaction of the medium's magnetization with external static or time-varying magnetic fields forms the basis for a wealth of applications of optically pumped media in fundamental and applied research fields [2].

For sensitive applications one wishes to ensure a long lifetime of the produced magnetization, by suppressing spin relaxation due to atom-container wall or atom-atom collisions. The former can be strongly suppressed by addition of a buffer gas or by antirelaxation wall coatings. The latter cause a more severe problem, in particular in alkali-metal vapors, where any win in signal strength from an increased vapor pressure is counteracted by an increase of the atomic collision rate and the ensuing relaxation from spin-exchange collisions. While spin-exchange relaxation can be suppressed in high-density alkali-metal vapors [3], it remains a main sensitivity limiting

\footnotetext{
*yongqi.shi@unifr.ch

†antoine.weis@unifr.ch
}

factor in room temperature alkali-metal vapors in (vacuum) wall-coated cells.

The initial goal of the study presented in this paper was the experimental verification of model calculations describing the decrease of the atomic absorption coefficient $\kappa_{0}$ with increasing light power $P$. We will show that under the assumption of isotropic spin-relaxation one can derive algebraic expressions for $\kappa_{0}(P)$ from rate equations. Obtaining poor agreement between the predicted and recorded dependences, we were led to extend our theoretical model by including (nonisotropic) spin-exchange relaxation. On the modeling side this implied that the rate equations could no longer be solved analytically, but required numerical procedures. We find that this extended model for $\kappa_{0}(P)$ yields an excellent description of the experimental results for all four hyperfine components of the ${ }^{133} \mathrm{Cs} D_{1}$ transition under pumping with linearly and circularly polarized light. The deployed fitting procedure yields values of the absorption coefficients, the optical pumping saturation power, and the ratio of the spin-exchange relaxation rate to the isotropic relaxation rate, which are all in very good agreement with theoretical expressions on an absolute scale.

\section{EXPERIMENTS}

\section{A. Apparatus}

The experimental setup is shown in Fig. 1. We use light from a fiber-coupled single-mode external-cavity diode laser (Toptica, model DL pro) operated at $\sim 894 \mathrm{~nm}$ near the Cs $6 S_{1 / 2} \rightarrow 6 P_{1 / 2}$ transition ( $D_{1}$ line). A small percentage of the light is directed into a Doppler-free saturated absorption frequency lock permitting one to actively lock the laser frequency to one of the four Cs $D_{1}$ hyperfine transitions.

The main part of the beam passes through a fiber-integrated electro-optic modulator (EOM) (Jenoptik, model AM905b). A small fraction of the light transmitted by the EOM is detected 


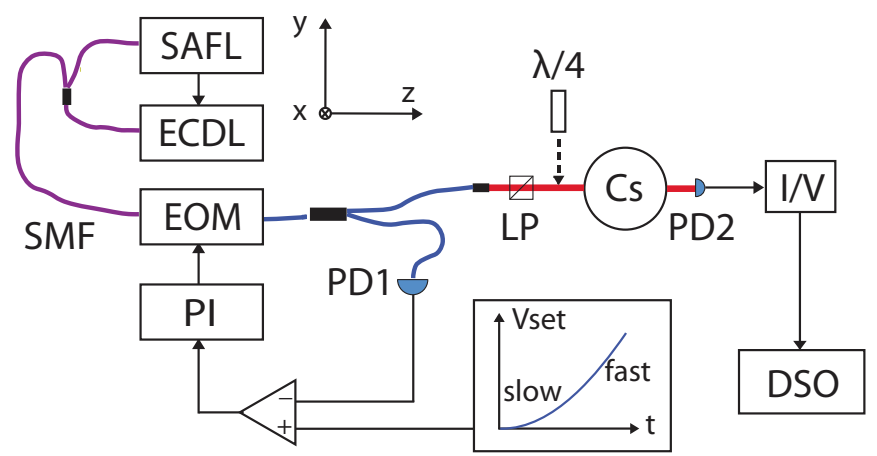

FIG. 1. Schematic of the experimental setup: ECDL, externalcavity diode laser; SAFL, saturated absorption frequency lock; SMF, single-mode fiber; EOM, electro-optic modulator; PI, proportionalintegral amplifier; PD1,2, photodiode; LP, linear polarizer; $\lambda / 4$, quarter waveplate; $I / V$, current-voltage converter; DSO, digital storage oscilloscope; and Cs, Cs vapor cell. The magnetic shield and the coils are not shown.

by a photodiode (PD1, Thorlabs, model DET36A/M). Comparison of the transimpedance-amplified photodiode signal with a variable voltage $V_{\text {set }}$ allows stabilizing the power $P$ transmitted by the EOM to a value $P \propto V_{\text {set }}$ using a feedback loop with proportional-integral control electronics [4]. More importantly, the feedback control allows scanning the power $P(t)$ of the laser light delivered to the experiment in an arbitrary manner by applying a suitable voltage $V_{\text {set }}(t)$ to the set value input of the feedback circuit. The polarization of the laser light can be changed from linearly polarized light to circularly polarized by insertion of a quarter waveplate $(\lambda / 4)$ after the polarizer.

The Cs vapor cell is an evacuated spherical Pyrex cell [29(1) mm inner diameter] with a paraffin-coated inner wall, which efficiently reduces the spin-relaxation rates due to wall collisions [5]. The cell contains a saturated vapor of Cs atoms that is in thermodynamic equilibrium (at room temperature) with a droplet of solid cesium contained in a reservoir stem, connected to the cell by an approximately $0.7-\mathrm{mm}$-diam capillary.

The laser beam from the single-mode fiber has a welldefined transverse Gaussian intensity distribution $I(r)=$ $I_{0} \exp \left(-2 r^{2} / w^{2}\right)$ with $w \approx 2 \mathrm{~mm}$ at the cell position. It propagates through the cell along the $z$ direction and the transmitted power is measured by a photodiode (PD2, Hamamatsu, model S6775-01) followed by a current-voltage converter (FEMTO, model DLPCA-200) whose voltage signal is recorded by a 16-bit digital storage oscilloscope (TiePie, model HS5).

The cell is mounted in the center of a three-layer cylindrical $\mu$-metal shield (not shown in the figure), in which the $B_{x}, B_{y}$, and $B_{z}$ components of the remnant field (approximately equal to several $\mathrm{nT}$ ) are measured and then compensated using the methods described in Refs. [6,7]. For this measurement and/or compensation we use three pairs of well-calibrated coils (not shown in the figure). After nulling all field components we apply a static holding field $B_{0}$ of approximately $40 \mathrm{nT}$ for the measurements proper. For measurements with circularly polarized light, $\vec{B}_{0}$ is oriented along the $\vec{k}$ vector of the beam

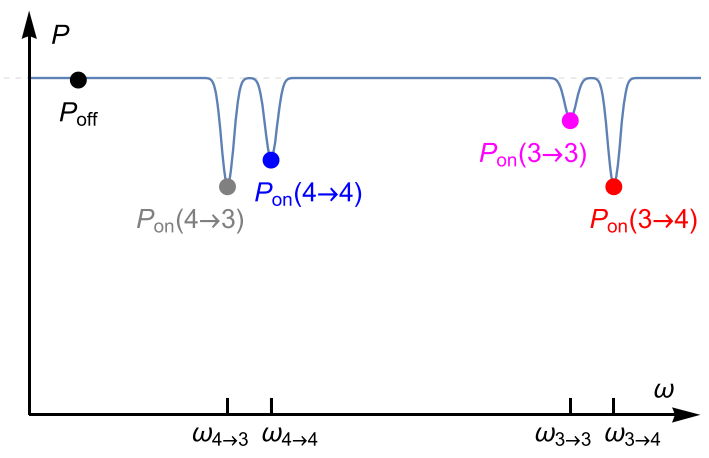

FIG. 2. Transmission spectrum showing the hyperfine structure of the Cs $D_{1}$ transition in a paraffin-coated vapor cell. The colored dots mark the frequencies at which the laser was set for the power scans.

( $z$ direction), while for measurements with linearly polarized light it is oriented along the light polarization, i.e., the $y$ direction. The purpose of the holding field is to stabilize the spin polarization produced by optical pumping in both cases. It is well known $[6,8]$ that this stabilization requires the Larmor frequency of the holding field to obey $\omega_{L}=\gamma_{F} B_{0} \gg \gamma_{1}$, where $\gamma_{F} \approx 3.5 \mathrm{~Hz} / \mathrm{nT}$ is the Cs ground state's gyromagnetic ratio and $\gamma_{1}$ the longitudinal spin-relaxation time of that state.

\section{B. Data recording}

Figure 2 shows the dependence of the transmitted laser power $P$ on the laser frequency $\omega$, when the latter is swept over the four hyperfine components $F_{g} \rightarrow F_{e}$ of the Cs $D_{1}$ transition. During this recording the power entering the cell was stabilized using the procedure discussed above, hence the flat off-resonance background $P_{\text {off }}$. The quantity of interest, i.e., the on-resonance absorption coefficient $\kappa_{0}$, is contained in the contrast, i.e., the relative amplitudes of the absorption dips.

We recall that the goal of the study was the measurement of the power dependence of $\kappa_{0}$ of the four transitions. In principle, this can be achieved by recording spectra like the one shown in Fig. 2 for a set of discrete settings of the laser power. However, we found the following method to be much more efficient for achieving the set goal: Because of the power stabilization procedure, the off-resonance power level $P_{\text {off }}$ is independent of the laser frequency. In order to extract the absorption coefficient, it is therefore sufficient to know the transmitted power at the five frequencies marked by dots in Fig. 2. While the four on-resonance measurements $P_{\text {on }}\left(F_{g} \rightarrow F_{e}\right)$ are done with the laser frequency actively locked to the corresponding transition, the off-resonance measurement was done by red detuning the frequency with respect to the $4 \rightarrow 3$ transition by approximately $1 \mathrm{GHz}$. For each set frequency we vary the incident laser power over the range of $1-10 \mu \mathrm{W}$ by applying a suitable time-dependent voltage $V_{\text {set }}(t)$ to the set input of the power stabilization system. Here $V_{\text {set }}(t)$ was given the shape of a quarter period of a sine wave (on a suitable offset, as shown schematically in Fig. 1), thus ensuring a slower scan speed at low power levels. This condition is required by the fact that a low power level $P$ implies a corresponding low optical pumping rate $\gamma_{\text {pump }} \propto P_{0}$ and hence a correspondingly longer time for achieving steady-state polarization conditions. 

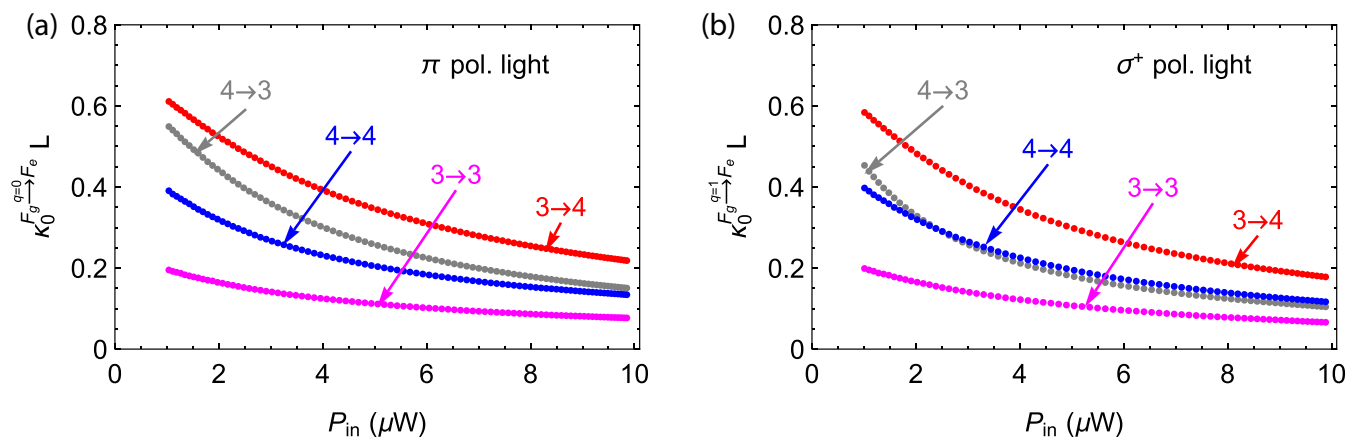

FIG. 3. Experimental measurements of the dependence of the optical thickness $\kappa_{0}^{F_{g}} \stackrel{q}{\rightarrow} F_{e} L$ on the power $P_{\text {in }}$ for all four hyperfine transitions of the Cs $D_{1}$ line. Data were recorded with both (a) linearly polarized and (b) circularly polarized light.

A 250-s time duration for a complete power scan was found sufficient for ensuring undistorted $P_{\text {off } / o n}(t)$ dependences.

\section{Extracting $\kappa_{0} L$}

The light power of a laser beam traversing the Cs vapor cell is given by

$$
P(\delta \omega)=P_{\text {in }} \mathcal{T} e^{-\kappa(\delta \omega) L}=P_{0} \mathcal{T}^{2} e^{-\kappa(\delta \omega) L},
$$

where $P_{\text {in }}$ is the power inside the cell, $P_{0}$ the power before the cell, $\mathcal{T}=0.92(1)$ the (power) transmission coefficient of each cell window, $L$ the length of the traversed atomic medium, and $\delta \omega=\omega_{F_{g} \rightarrow F_{e}}-\omega$ the detuning of the laser frequency $\omega$ from an atomic transition frequency $\omega_{F_{g} \rightarrow F_{e}}$. The absorption coefficient can be expressed as

$$
\kappa(\delta \omega)=\kappa_{0} \mathcal{L}(\delta \omega),
$$

where $\kappa_{0}=\kappa(\delta \omega=0)$ is the peak absorption coefficient and $\mathcal{L}(\delta \omega)$ the spectral line shape function (Lorentzian, Doppler, Voigt, or other profile), normalized such that $\mathcal{L}(0)=1$.

Denoting the off-resonance transmitted power by

$$
P_{\text {off }} \equiv P(\delta \omega \gg \Gamma)=P_{\text {in }} \mathcal{T},
$$

where $\Gamma$ is the resonance linewidth, and the on-resonance power by

$$
P_{\text {on }} \equiv P(\delta \omega=0)=P_{\text {in }} \mathcal{T} e^{-\kappa_{0} L},
$$

one sees that the on-resonance optical thickness is given by

$$
\kappa_{0} L=-\ln \frac{P_{\text {on }}}{P_{\text {off }}} .
$$

Following the procedure described above, we have measured the $P_{\text {on }}\left(P_{\text {in }}\right)$ dependences with the laser frequency set to the dotted values in Fig. 2. Using Eq. (5) we have then extracted the four $\kappa_{0}^{F_{g} \stackrel{q}{\rightarrow} F_{e}}\left(P_{\text {in }}\right) L$ dependences. We performed these measurements both with linearly $(q=0)$ and with circularly $(q=1)$ polarized light. The experimental results are shown in Fig. 3 and illustrate the well-known decrease of the absorption (corresponding to an increase of the transmission) under the effect of optical pumping.

\section{THEORETICAL MODELING}

In this section we describe our approach to the theoretical modeling of the dependences shown in Fig. 3. We will first show that under the assumption of isotropic spin relaxation in the ground state one can derive algebraic expressions for the four $\kappa_{0}^{F_{g} \stackrel{q}{\rightarrow} F_{e}}\left(P_{\text {in }}\right)$ dependences.

We note that the cell's sidearm (reservoir) points in a radial direction with respect to the laser beam's propagation direction, thus breaking rotational symmetry. Since many wall collisions randomize the directions of the atoms' trajectories, before their escape into the reservoir, the relaxation process associated with this loss mechanism can be assumed to be isotropic. However, we find that the assumption of isotropic relaxation gives rather poor agreement with the experimental findings.

As argued in Ref. [5], we know that spin relaxation in the deployed paraffin-coated cell has a strong contribution from spin-exchange collisions, which, by their nature yield a nonisotropic (i.e., $F$ - and $m$-dependent) relaxation. In consequence, we have extended the theoretical model in order to take both isotropic and spin-exchange collisions into account.

\section{A. Setting up rate equations}

We describe the atomic vapor in terms of the populations $p_{F, m}$ of the two hyperfine levels $F=3,4$ of the $6 S_{1 / 2}$ cesium ground state. These populations are represented in terms of a population vector $\vec{p}$ with $16=(2 I+1)\left(2 J_{g}+1\right)$ components $\left\{p_{3,-3}, \ldots, p_{3,+3}, p_{4,-4}, \ldots, p_{4,+4}\right\}$. We neglect the population difference due to the finite Boltzmann factor $\exp \left(-E_{\mathrm{hf}} / k_{\mathrm{B}} T\right)$, $E_{\mathrm{hf}}$ being the hyperfine splitting, so that all populations are equal to $1 / 16$ when the medium is not spin polarized.

We consider laser light that is resonant with a given hyperfine transition $\left|F_{g}\right\rangle \equiv\left|6 S_{1 / 2}, F_{g}\right\rangle \rightarrow\left|F_{e}\right\rangle \equiv\left|6 P_{1 / 2}, F_{e}\right\rangle$ of the Cs $D_{1}$ line. Optical pumping will redistribute the groundstate populations by subsequent photon absorption-reemission cycles. Since the maximum photon absorption rate $\gamma_{\mathrm{abs}}$ in our experiments is less than $(2 \pi) \times 100 \mathrm{~Hz}$ (for $10 \mu \mathrm{W}$, as shown in Sec. VB) while the spontaneous photon emission from the excited state occurs at a rate $\gamma_{\mathrm{em}}$ of several $\mathrm{MHz}$, the pump rate $\gamma_{\text {pump }}\left(\left|F_{g}, m_{g}\right\rangle \rightarrow\left|F_{e}, m_{e}\right\rangle\right)$ can be assumed to be equal to the absorption rate $\gamma_{\text {pump }} \propto \gamma_{\text {abs }}$, given by

$$
\gamma_{F_{e}, m_{e} ; F_{g}, m_{g}}^{\text {pump }}=\gamma_{\mathrm{abs}}^{F_{g}, m_{g} \stackrel{q}{\rightarrow} F_{e}, m_{e}} .
$$

Note that here and below we use ordered indices that are to be read as $X_{i ; j} \equiv X_{\text {to;from }}$. As shown in Appendix A, the rate at which light is absorbed on a given sublevel transition 


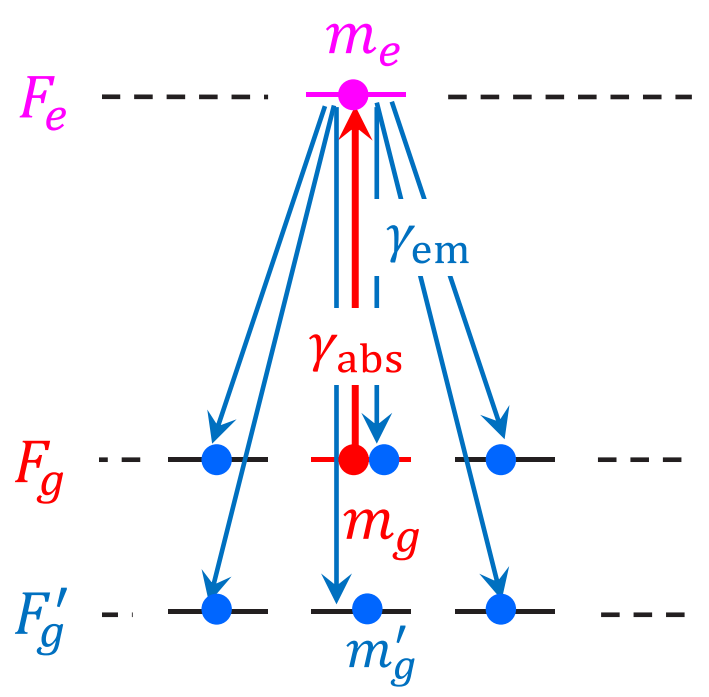

FIG. 4. Hyperfine and magnetic sublevel structure used to discuss the optical pumping rate equations.

$\left|F_{g}, m_{g}\right\rangle \rightarrow\left|F_{e}, m_{e}\right\rangle$ in a Doppler-broadened medium is given by

$$
\gamma_{\mathrm{abs}}^{F_{g}, m_{g} \stackrel{q}{\rightarrow} F_{e}, m_{e}}=c_{F_{e}, m_{e} ; F_{g}, m_{g}}^{(q)} \Gamma_{\mathrm{abs}},
$$

where

$$
\begin{aligned}
c_{F_{e}, m_{e} ; F_{g}, m_{g}}^{(q)}= & \left(2 F_{g}+1\right)\left(2 F_{e}+1\right) \\
& \times\left(\begin{array}{ccc}
F_{e} & 1 & F_{g} \\
-m_{e} & q & m_{g}
\end{array}\right)^{2}\left\{\begin{array}{ccc}
J_{e} & J_{g} & 1 \\
F_{g} & F_{e} & I
\end{array}\right\}^{2}
\end{aligned}
$$

and

$$
\Gamma_{\mathrm{abs}}=\frac{4 \pi^{3 / 2} \alpha|r|^{2}}{\hbar \Gamma_{D}} \frac{P_{\mathrm{in}}}{S_{\mathrm{eff}}} .
$$

Here $P_{\text {in }}$ is the power carried by the laser beam inside the cell, $S_{\text {eff }}$ is the beam's effective cross section, $\Gamma_{D}$ is the Doppler width, introduced in Appendix A 1, and $|r|=\left|\left\langle 6 P_{1 / 2}\|r\| 6 S_{1 / 2}\right\rangle\right|$ is the reduced electric dipole matrix element of the $D_{1}$ transition.

The rate at which ground-state populations $p_{F_{g}, m_{g}}$ are transferred to excited states $\left|F_{e}, m_{e}\right\rangle$ (processes marked by the upward red arrow in Fig. 4) can be expressed in terms of a depopulation matrix $\mathbb{D}$, whose matrix elements are given by

$$
\gamma_{F_{e}, m_{e} ; F_{g}, m_{g}}^{\text {pump }}=\mathbb{D}_{F_{e}, m_{e} ; F_{g}, m_{g}} \Gamma_{\mathrm{abs}}=c_{F_{e}, m_{e} ; F_{g}, m_{g}}^{(q)} \Gamma_{\mathrm{abs}} .
$$

As shown in Fig. 4 for the case of linearly polarized light $(q=0)$, each pumping cycle (i.e., absorption-reemission cycle) will transfer, in general, populations from a given state $\left|F_{g}, m_{g}\right\rangle$ (marked by a red dot in the figure) to six adjacent sublevels (marked by blue dots).

The elements of the repopulation matrix $\mathbb{R}$ describing the rates at which the ground-state levels $\left|F_{g}^{\prime}, m_{g}^{\prime}\right\rangle$ are repopulated after one pumping cycle starting from $\left|F_{g}, m_{g}\right\rangle$ are given by

$$
\begin{aligned}
\gamma_{F_{g}^{\prime}, m_{g}^{\prime} ; F_{g}, m_{g}}^{\text {repop }} & =\mathbb{R}_{F_{g}^{\prime}, m_{g}^{\prime} ; F_{g}, m_{g}} \Gamma_{\mathrm{abs}} \\
& =w_{F_{g}^{\prime}, m_{g}^{\prime} ; F_{e}, m_{e}} c_{F_{e}, m_{e} ; F_{g}, m_{g}} \Gamma_{\mathrm{abs}},
\end{aligned}
$$

where the probabilities $w$ are given by

$$
\begin{aligned}
w_{F_{g}^{\prime}, m_{g}^{\prime} ; F_{e}, m_{e}}= & 2\left(2 F_{e}+1\right)\left(2 F_{g}^{\prime}+1\right) \\
& \times\left(\begin{array}{ccc}
F_{g}^{\prime} & 1 & F_{e} \\
-m_{g}^{\prime} & m_{g}^{\prime}-m_{e} & m_{e}
\end{array}\right)^{2}\left\{\begin{array}{ccc}
J_{e} & J_{g} & 1 \\
F_{g}^{\prime} & F_{e} & I
\end{array}\right\}^{2} .
\end{aligned}
$$

The forefactors in Eq. (12) ensure that the probabilities are normalized such that $\sum_{F_{g}^{\prime}, m_{g}^{\prime}} w_{F_{g}^{\prime}, m_{g}^{\prime} ; F_{e}, m_{e}}=1$ for any sublevel $\left|F_{e}, m_{e}\right\rangle$. Defining a (dimensionless) pump operator $\mathbb{P}=$ $-\mathbb{D}+\mathbb{R}$, the rate equations governing the temporal evolution of the population vector are given by

$$
\dot{\vec{p}}=\mathbb{P} \Gamma_{\text {abs }} \vec{p}-\gamma_{1} \mathbb{1}\left(\vec{p}-\vec{p}^{\mathrm{eq}}\right),
$$

where $\mathbb{1}$ is the unit operator and we have assumed that in the absence of light $\left(P_{\text {in }}=0 \rightarrow \Gamma_{\text {abs }}=0\right)$ the ground-state populations relax isotropically (i.e., independently of $F_{g}$ and $m_{g}$ ) at a rate $\gamma_{1}$ towards the 16 equilibrium populations $\vec{p}^{\text {eq }}=$ $\{1 / 16,1 / 16, \ldots, 1 / 16\}$.

\section{B. Solving the rate equations}

For $\mathbb{P} \neq 0$, the ground-state sublevel populations evolve towards steady-state populations $\vec{p}_{\text {ss }}$ that are defined by the condition $\dot{\vec{p}}=0$. The 16 -component steady-state population vector is thus obtained by solving the system

$$
\sum_{j}\left(\mathbb{R}_{i j} \Gamma_{\mathrm{abs}}-\mathbb{D}_{i j} \Gamma_{\mathrm{abs}}-\delta_{i j} \gamma_{1}\right) p_{j}^{\mathrm{ss}}=\gamma_{1} p_{i}^{\mathrm{eq}},
$$

or equivalently

$$
\sum_{j}\left(\mathbb{R}_{i j} x-\mathbb{D}_{i j} x-\delta_{i j}\right) p_{j}^{\mathrm{ss}}=p_{i}^{\mathrm{eq}},
$$

of 16 algebraic equations, in which $i$ and $j$ denote the quantum numbers of the 16 magnetic sublevels of the ground state. In Eq. (15) we have introduced the dimensionless optical pumping saturation parameter

$$
x \equiv \frac{\Gamma_{\mathrm{abs}}}{\gamma_{1}}=\frac{4 \pi^{3 / 2} \alpha|r|^{2}}{\hbar \Gamma_{D} \gamma_{1}} \frac{P_{\mathrm{in}}}{S_{\mathrm{eff}}} \equiv \frac{P_{\mathrm{in}}}{P_{\mathrm{sat}}},
$$

where

$$
P_{\mathrm{sat}}=\frac{\hbar \Gamma_{D} \gamma_{1} S_{\mathrm{eff}}}{4 \pi^{3 / 2} \alpha|r|^{2}}
$$

is the optical pumping saturation power. Writing out the expression (15) explicitly yields

$$
\begin{aligned}
& \sum_{F_{g}=3}^{4} \sum_{m_{g}=-F_{g}}^{+F_{g}}\left[\delta_{F_{g}, \tilde{F}_{g}}\left(w_{F_{g}^{\prime}, m_{g}^{\prime} ; F_{e}, m_{e}} c_{F_{e}, m_{e} ; F_{g}, m_{g}}^{(q)}-c_{F_{e}, m_{e} ; F_{g}, m_{g}}^{(q)}\right) x\right. \\
& \left.\quad-\delta_{F_{g}^{\prime}, m_{g}^{\prime} ; F_{g}, m_{g}}\right] p_{F_{g}, m_{g}}^{\mathrm{ss}}=p_{F_{g}^{\prime}, m_{g}^{\prime}}^{\mathrm{eq}}
\end{aligned}
$$

where $\left|\tilde{F}_{g}\right\rangle$ is the ground state to which the laser light couples. The 16 rate equations can be solved algebraically using, e.g., Mathematica [9], yielding rational expressions for the steady-state populations in the states $\left|F_{g}, m_{g}\right\rangle$ produced by optical pumping on the $\left|F_{g}\right\rangle \rightarrow\left|F_{e}\right\rangle$ transition by $q$-polarized 

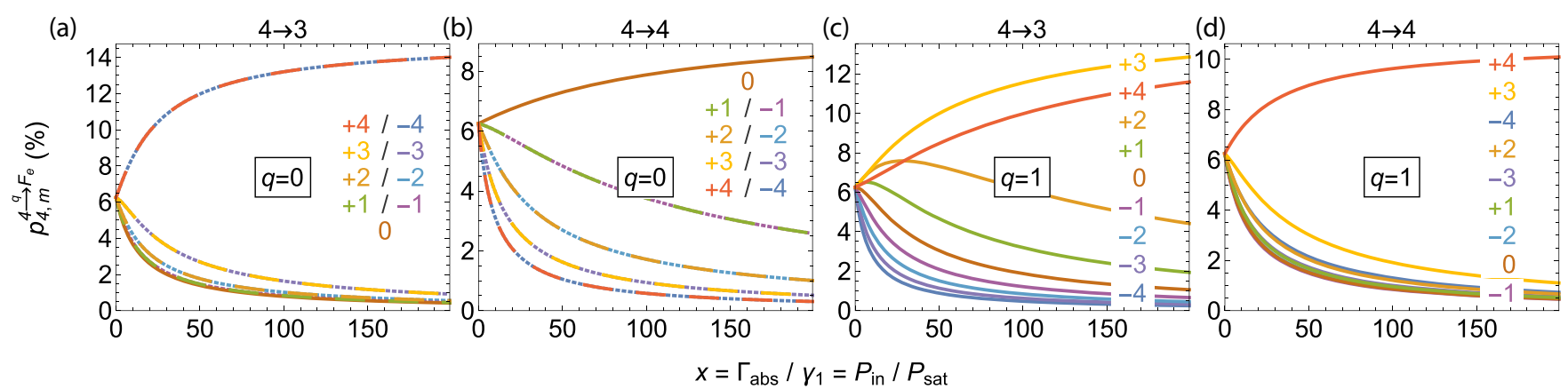

FIG. 5. Anticipated power dependence of the sublevel populations $p_{4, m}$ in the $F_{g}=4$ state when pumped on the $4 \rightarrow 3$ and $4 \rightarrow 4$ transitions with linearly $(q=0)$ and circularly $(q=1)$ polarized light, assuming isotropic relaxation. The numbers on the right in each graph represent the $m_{g}$ values, in the same top-to-bottom order as the curves. We note that for $q=0$, the (dashed) lines with a given $m_{g}$ overlap the (dotted) lines with $-m_{g}$.

light

$$
p_{F_{g}, m_{g}}^{\stackrel{F_{g}}{\stackrel{q}{\rightarrow}} F_{e}}(x)=\mathcal{A} \frac{\sum_{n} a_{n} x^{n}}{\sum_{n} b_{n} x^{n}},
$$

where $\mathcal{A}, a_{n}$, and $b_{n}$ depend on $F_{g}, F_{e}, m_{g}$, and $q$.

Figures 5 and 6 show the evolution of the steady-state populations with the parameter $x=P_{\text {in }} / P_{\text {sat }}$. Note that only populations of the ground state $F_{g}$ to which the pumping laser couples are shown.

Let us discuss the graphs in Fig. 5, which represent the populations of the $\left|F_{g}=4, m_{g}\right\rangle$ states after pumping on the $F_{g}=$ $4 \rightarrow F_{e}=3,4$ transitions. One can see that populations are transferred preferentially to the $\left|F_{g}=4, m_{g}= \pm 4\right\rangle$ states and to the $\left|F_{g}=4, m_{g}=0\right\rangle$ state when pumping with linearly polarized $(q=0)$ light on the $F_{g}=4 \rightarrow F_{e}=3$ and $F_{g}=4 \rightarrow$ $F_{e}=4$ transitions, respectively [Figs. 5(a) and 5(b)]. These so-called dark states do not absorb linearly polarized light, a manifestation of electromagnetically induced transparency (EIT). The corresponding dark states under pumping with circularly polarized $(q=1)$ light are the $\left|F_{g}=4, m_{g}=3,4\right\rangle$ and $\left|F_{g}=4, m_{g}=4\right\rangle$ states, respectively [Figs. 5(c) and 5(d)].

The populations of the $\left|F_{g}=3, m_{g}\right\rangle$ states shown in Fig. 6 exhibit a peculiar feature. While pumping on the $3 \rightarrow 3$ transition with linearly or circularly polarized light produces dark states [Figs. 6(a) and 6(c)], pumping on the $3 \rightarrow 4$ transitions completely empties the populations in the $\left|F_{g}=3\right\rangle$ state, the emptying being more efficient under pumping with linearly polarized light [Figs. 6(b) and 6(d)]. These transitions are thus useful for repumping experiments.

The above discussion shows that the medium becomes transparent, for all presented cases, in the high power limit $(x \rightarrow \infty)$, but that the underlying mechanism may be quite different, i.e., creation of dark state(s) versus complete level depletion. As shown in Ref. [10], optical pumping on a closed $F \rightarrow F+1$ transition transfers atoms to a state whose absorption coefficient is larger than the one of the unpolarized state $F$, an effect known as electromagnetically induced absorption (EIA). The fact that EIA does not occur for the $3 \rightarrow 4$ transition here is due to the open character of the transition, i.e., the loss of atoms to the $F=4$ ground state that does not couple to the light. We note that the rate equation model is valid only if optical saturation can be neglected, i.e., as long as $\Gamma_{\mathrm{abs}} \ll \gamma_{\mathrm{em}}$, a condition that is well fulfilled in our case as discussed above.

\section{Peak absorption coefficients}

With the state populations known, we can then calculate the power dependence of the peak absorption coefficient for each hyperfine transition following Eqs. (A10) and (A11),

$$
\kappa_{0}^{F_{g} \stackrel{q}{\rightarrow} F_{e}}=\sum_{m_{g}=-F_{g}}^{+F_{g}} p_{F_{g}, m_{g}}^{F_{g} \stackrel{q}{\rightarrow} F_{e}} c_{F_{e}, m_{e} ; F_{g}, m_{g}}^{(q)} \tilde{C}_{\kappa},
$$

(a)

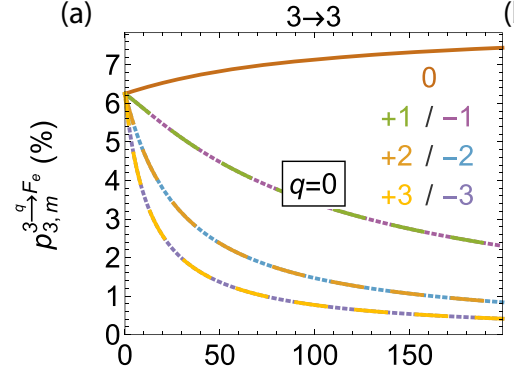

(b)

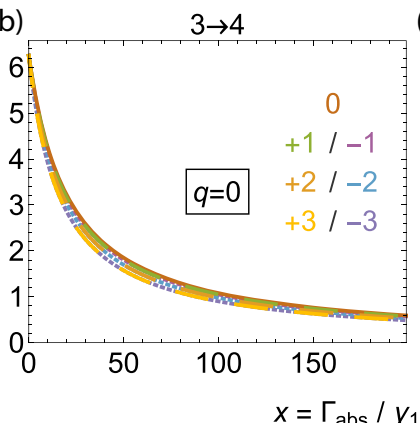

(c)

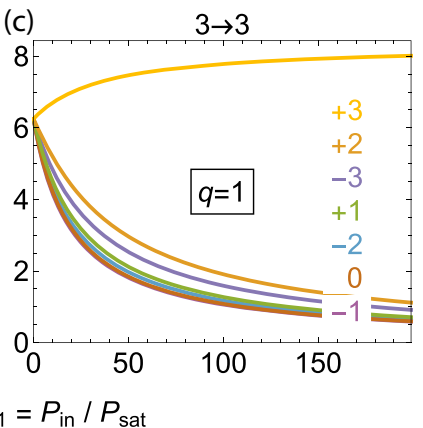

(d)

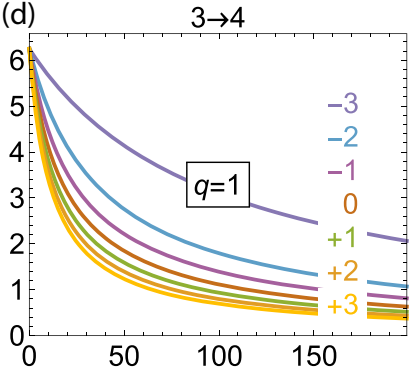

FIG. 6. Anticipated power dependence of the sublevel populations $p_{3, m}$ in the $F_{g}=3$ state when pumped on the $3 \rightarrow 3$ and $3 \rightarrow 4$ transitions with linearly $(q=0)$ and circularly $(q=1)$ polarized light, assuming isotropic relaxation. The numbers on the right in each graph represent the $m_{g}$ values, in the same top-to-bottom order as the curves. As in Fig. 5, the (dashed) lines with a given $m_{g}$ overlap the (dotted) lines with $-m_{g}$ for $q=0$. 

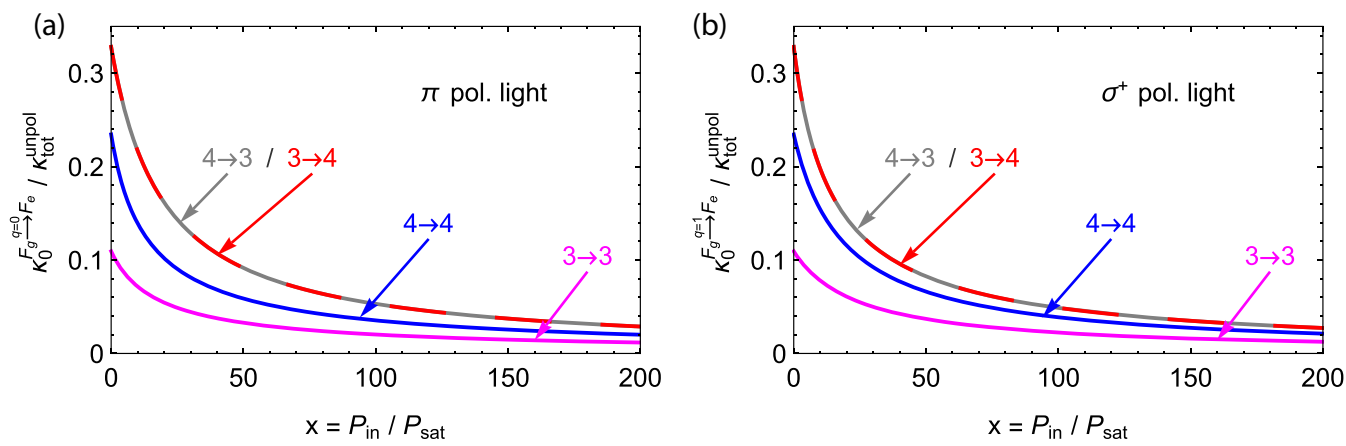

FIG. 7. Anticipated dependence of the (normalized) peak absorption coefficients on the dimensionless parameter $x=\Gamma_{\text {abs }} / \gamma_{1}=P_{\text {in }} / P_{\text {sat }}$ given by the analytical expressions [Eq. (22)] derived from rate equations, assuming isotropic population relaxation for pumping with (a) linearly polarized light and (b) circularly polarized light.

with

$$
\tilde{C}_{\kappa}=4 \pi \alpha \sqrt{\frac{\pi M_{\mathrm{Cs}} c^{2}}{2 k_{B} T}}|r|^{2} N_{\mathrm{Cs}} .
$$

Since we have explicit algebraic expression for the populations

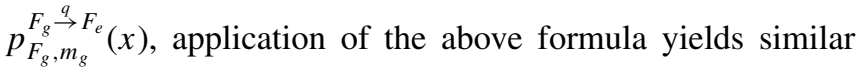
algebraic rational expressions for the absorption coefficients

$$
\frac{\kappa_{0}^{F_{g}} \stackrel{q}{\rightarrow} F_{e}(x)}{\kappa_{\text {tot }}^{\text {unpol }}}=\mathcal{B} \frac{\sum_{n} \mathcal{N}_{n} x^{n}}{\sum_{n} \mathcal{D}_{n} x^{n}},
$$

where $\kappa_{\text {tot }}^{\text {unpol }}=\tilde{C}_{\kappa} / 6$, as shown in Appendix A. Numerical values of the (integer) coefficients $\mathcal{B}, \mathcal{N}_{n}$, and $\mathcal{D}_{n}$ that depend on $F_{g}, F_{e}$, and $q$ are beyond the scope of the present work.

Expressing all $\kappa_{0}^{F_{g} \stackrel{q}{\rightarrow} F_{e}}(x)$ in units of $\kappa_{\text {tot }}^{\text {unpol }}$ yields, for the individual absorption coefficients in the limit $x \rightarrow 0$,

$$
\begin{aligned}
& \frac{\kappa_{0}^{4 \rightarrow 4}(0)}{\kappa_{\text {tot }}^{\text {unpol }}}=\frac{21}{64}, \quad \frac{\kappa_{0}^{4 \rightarrow 3}(0)}{\kappa_{\text {tot }}^{\text {unpol }}}=\frac{15}{64}, \\
& \frac{\kappa_{0}^{3 \rightarrow 3}(0)}{\kappa_{\text {tot }}^{\text {unpol }}}=\frac{7}{64}, \quad \frac{\kappa_{0}^{3 \rightarrow 4}(0)}{\kappa_{\text {tot }}^{\text {unpol }}}=\frac{21}{64},
\end{aligned}
$$

independently of the light polarization $q$. The graphs in Fig. 7 show the dependence of the peak absorption coefficients (in units of $\kappa_{\mathrm{tot}}^{\text {unpol }}$ ) for the four hyperfine components on the dimensionless parameter $x$ that is proportional to laser power. The results are shown for pumping with linearly polarized light [Fig. 7(a)] and with circularly polarized light [Fig. 7(b)]. All coefficients vanish for $x \rightarrow \infty$, a trivial manifestation of the well-studied phenomenon of EIT.

The dependences have the specific property that the algebraic expressions for $\kappa_{0}^{4 \rightarrow 3}(x)$ and $\kappa_{0}^{3 \rightarrow 4}(x)$ are perfectly identical, for both $q=0$ and $q=1$. Comparison with the experimental recordings of Fig. 3 shows that the latter do not reflect this anticipated feature.

It was known from our previous study [5] that, even at room temperature, spin relaxation in the deployed paraffin-coated cell has a strong (or even dominant) contribution from spinexchange collisions which are nonisotropic, i.e., $F_{g}$ and $m_{g}$ dependent. It was thus natural to assume that the failure of the simple rate equations in reproducing the experimental data was due to the simplifying assumption of isotropic relaxation. In the following section we will describe how we have extended the above model by implementing spin-exchange relaxation.

\section{Spin-exchange relaxation}

Spin-exchange relaxation is a process in which colliding ground-state atoms in different hyperfine Zeeman states $\left|F_{g}, m_{g}\right\rangle$ and $\left|F_{g}^{\prime}, m_{g}^{\prime}\right\rangle$ exchange their quantum numbers such that $m_{g}+m_{g}^{\prime}$ is conserved. In the density-matrix formalism, spin-exchange relaxation can be expressed as $\dot{\rho}=-\gamma_{\text {se }} \varpi_{\text {se }}$, where $\gamma_{\mathrm{se}}$ is the spin-exchange rate and $\mathbb{\nabla}_{\mathrm{se}}$ the dimensionless spin-exchange operator

$$
\mathbb{\square}_{\mathrm{se}}=\frac{3}{4} \rho-\overrightarrow{\mathbb{S}} \cdot \rho \overrightarrow{\mathbb{S}}-\langle\overrightarrow{\mathbb{S}}\rangle \cdot(\{\overrightarrow{\mathbb{S}}, \rho\}-2 i \overrightarrow{\mathbb{S}} \times \rho \overrightarrow{\mathbb{S}})
$$

that is derived and discussed in Refs. [11-14]. We note that the spin-exchange rate has a strong temperature dependence since $\gamma_{\mathrm{se}} \propto N_{\mathrm{Cs}}$. In Eq. (23), $\{\cdots\}$ denotes the anticommutator, and $\langle\overrightarrow{\mathbb{S}}\rangle=\operatorname{tr}(\rho \overrightarrow{\mathbb{S}})$ is the (vector) spin polarization, i.e., the Bloch vector of the atomic medium. Appendix B gives explicit expressions for the matrix representation of the spin operators $\mathbb{S}_{x, y, z}$.

The operator form of the rate equations that take both isotropic and spin-exchange relaxation into account reads

$$
\dot{\vec{p}}=\mathbb{P} \Gamma_{\mathrm{abs}} \vec{p}-\gamma_{1} \mathbb{1}\left(\vec{p}-\vec{p}^{\mathrm{eq}}\right)-\gamma_{\mathrm{se}} \overrightarrow{\mathbb{G}}_{\mathrm{se}}(\vec{p}) .
$$

We have evaluated the matrix representation of $\mathbb{\Xi}_{\mathrm{se}}$ in the basis of the 16 sublevel populations, taking into account that $\rho$ has only diagonal elements (the sublevel populations). Moreover, since the problem at hand deals only with sublevel populations, we merely need the diagonal part $\left[\overrightarrow{\mathbb{G}}_{\mathrm{se}}\right]_{i}=\left[\mathbb{\mathbb { V }}_{\mathrm{se}}\right]_{i i}$ of $\mathbb{\varpi}_{\mathrm{se}}$. These diagonal elements contain terms that are linear and terms that are bilinear in the sublevel populations $p_{F_{g}, m_{g}}$ so that, when written in component form, the steady-state solutions $(\dot{\vec{p}}=0)$ of the rate equations are obtained by solving the nonlinear system of algebraic equations

$$
\sum_{j}\left(\mathbb{P}_{i j} x-\delta_{i j}\right) p_{j}^{\mathrm{ss}}-\varepsilon_{\mathrm{se}}\left[\overrightarrow{\mathbb{G}}_{\mathrm{se}}\left(\vec{p}^{\mathrm{ss}}\right)\right]_{i}=-p_{i}^{\mathrm{eq}},
$$

where $x=\Gamma_{\text {abs }} / \gamma_{1}=P_{\text {in }} / P_{\text {sat }}$ is the variable and $\varepsilon_{\mathrm{se}}=\gamma_{\mathrm{se}} / \gamma_{1}$ the relaxation parameter.

Because of the spin-exchange term's nonlinear dependence on the sublevel populations, the equations can no longer be solved algebraically. We obtain numerical solutions of Eq. (25) 
(a)

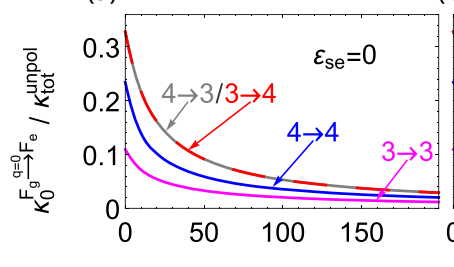

(b)

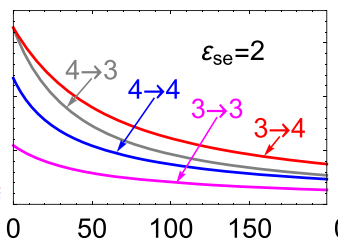

(c)

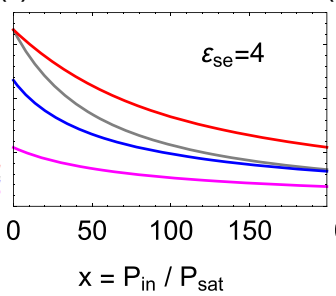

(d)

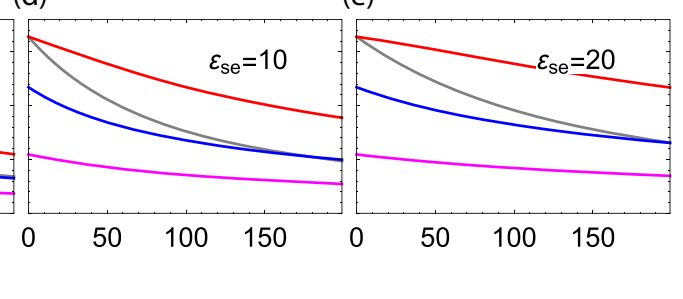

FIG. 8. Theoretical predictions of the power dependence of the normalized peak absorption coefficients $\kappa_{0}^{F_{g}} \stackrel{q=0}{\rightarrow} F_{e}(x) / \kappa_{\text {tot }}^{\text {unpol }}$ for pumping with linearly polarized light. Shown from left to right are increasing values of $\varepsilon_{\mathrm{se}}$, i.e., the ratio of the spin-exchange rate to the isotropic relaxation rate.

using Mathematica and use them for calculating the power dependence of the peak absorption coefficients following Eq. (20). The results are shown in Figs. 8 and 9 for various values of the spin-exchange parameter $\varepsilon_{\mathrm{se}}$. As anticipated, the overlapping curves for the $4 \rightarrow 3$ and $3 \rightarrow 4$ transitions found in the case of pure isotropic relaxation break up into two distinctive curves for $\varepsilon_{\mathrm{se}} \neq 0$.

\section{FITTING THE DATA}

Our model calculations yield power dependences of the form $\kappa_{0}^{F_{g} \stackrel{q}{\rightarrow} F_{e}}(x) / \kappa_{\text {tot }}^{\text {unpol }}$ as a function of the dimensionless variable $x=P_{\text {in }} / P_{\text {sat }}$. The experimental data, on the other hand, yield dependences of the optical thickness $\kappa_{0}^{F_{g} \stackrel{q}{\rightarrow} F_{e}} L$ as a function of the optical power $P_{\text {in }}$.

We have taken the following approach for fitting the theoretical (numerical) expressions to the data taken with circularly and with linearly polarized light, respectively: For each light polarization (i.e., $q=0$ and $q=1$ ), we calculate the absorption coefficients $\kappa_{0}^{F_{g}} \stackrel{q}{\rightarrow} F_{e}(x)$ of the four hyperfine transitions on a grid of 301 discrete $x$ values $x_{m}$ $\left(0 \leqslant x_{m} \leqslant 3000\right)$. We produce such sets for a series of 21 discrete $\varepsilon_{\mathrm{se}}$ values $\varepsilon_{\mathrm{se}}^{(n)}\left(0 \leqslant \varepsilon_{\mathrm{se}}^{(n)} \leqslant 20\right)$, yielding a twodimensional discrete set of $\kappa_{m, n}^{F_{g} \stackrel{9}{\rightarrow}} F_{e}\left(\varepsilon_{\text {se }}^{(n)} ; x_{m}\right) / \kappa_{\text {tot }}^{\text {unpol }}$ values. We next use an interpolation algorithm (based on twodimensional Hermite interpolation of order $3 \times 3$ ) to generate sets of continuous functions $\kappa_{\text {theor }}^{F_{g} \stackrel{q}{\rightarrow} F_{e}}\left(\varepsilon_{\mathrm{se}} ; x\right) / \kappa_{\text {tot }}^{\text {unpol }}$ that pass through the discrete $\kappa_{m, n}^{F_{g} \stackrel{q}{\rightarrow}} F_{e}\left(\varepsilon_{\mathrm{se}}^{(n)} ; x_{m}\right) / \kappa_{\text {tot }}^{\text {unpol }}$ points. We note that there are $8=2$ (polarizations) $\times 4$ (hyperfine transitions) such functions. Finally, we define fit functions $\kappa_{\mathrm{fit}}^{F_{g} \stackrel{q}{\rightarrow} F_{e}} L=$ $\tilde{A}^{q} \kappa_{\text {theor }}^{F_{g} \stackrel{q}{\rightarrow} F_{e}}\left(\tilde{\varepsilon}_{\mathrm{se}}^{q} ; P_{\text {in }} / \tilde{P}_{\text {sat }}^{q}\right) / \kappa_{\text {tot }}^{\text {unpol }}$ to fit the experimental data. For each polarization $q$, the three fit parameters, common to all four transitions, are an overall amplitude $\tilde{A}^{q}$, the saturation power $\tilde{P}_{\text {sat }}^{q}$, and the relaxation parameter $\tilde{\varepsilon}_{\text {se }}^{q}$.

\section{RESULTS AND DISCUSSION}

For each polarization $q$, we fit the four experimental curves in Fig. 3 simultaneously with one given set of parameters $\tilde{A}^{q}$, $\tilde{P}_{\text {sat }}^{q}$, and $\tilde{\varepsilon}_{\text {se }}^{q}$. Figure 10 shows the results for linearly polarized [Fig. 10(a)] and circularly polarized [Fig. 10(b)] light, obtained by varying the laser power between 1 and $10 \mu \mathrm{W}$. The model is seen to reproduce very well the experimental data. In the following, we discuss the numerical values of the three fit parameters that are shown in Table I.

\section{A. Fitted amplitude $\tilde{A}^{q}$}

According to our model (Appendix A 1), the global fitting parameter $\tilde{A}^{q}$ is defined as

$$
\tilde{A}^{q}=\kappa_{\mathrm{tot}}^{\mathrm{unpol}} L=\frac{2 \pi \alpha}{3}|r|^{2} \sqrt{\frac{\pi M_{\mathrm{Cs}} c^{2}}{2 k_{B} T}} N_{\mathrm{Cs}}(T) L,
$$

where $\alpha=\left(4 \pi \varepsilon_{0}\right)^{-1} e^{2} / \hbar c$ is the fine-structure constant. We compare this theoretical expression with the experimental $\tilde{A}^{q}$ values as follows. While the reduced matrix element $|r|=$ $4.4978 a_{0}$ ( $a_{0}$ being the Bohr radius) is known with high precision [15], we lack precise knowledge of the temperature $T$. Assuming a path length $L$ of 29(1) $\mathrm{mm}$ and using the expression from Ref. [16] for the temperature dependence of the number density $N_{\mathrm{Cs}}(T)$, we determine the temperature $T$, for which Eq. (26) reproduces the experimental $\tilde{A}^{q}$ values from Table I. We find $T=22.0(3){ }^{\circ} \mathrm{C}$ and $T=21.8(6){ }^{\circ} \mathrm{C}$ for the experiments with linearly polarized and circularly polarized light, respectively. These compatible results are consistent with (a) (b)

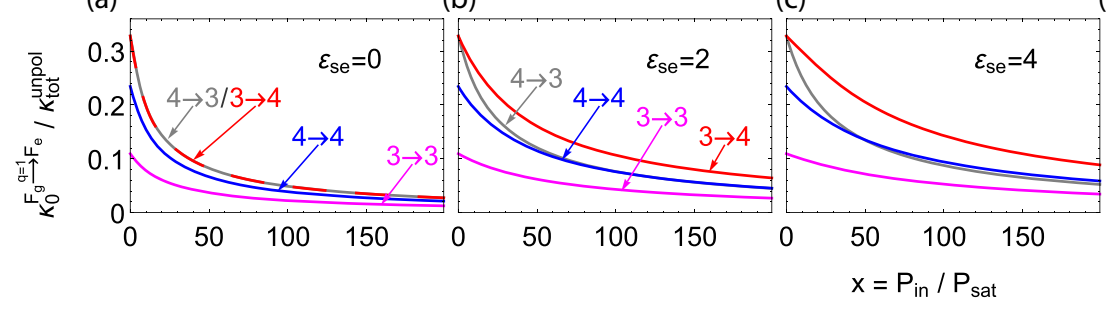

(d)

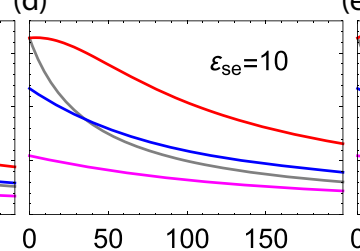

(e)

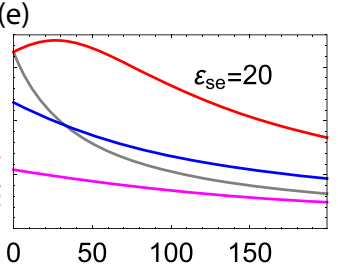

FIG. 9. Same as in Fig. 8 for pumping with circularly polarized light. 

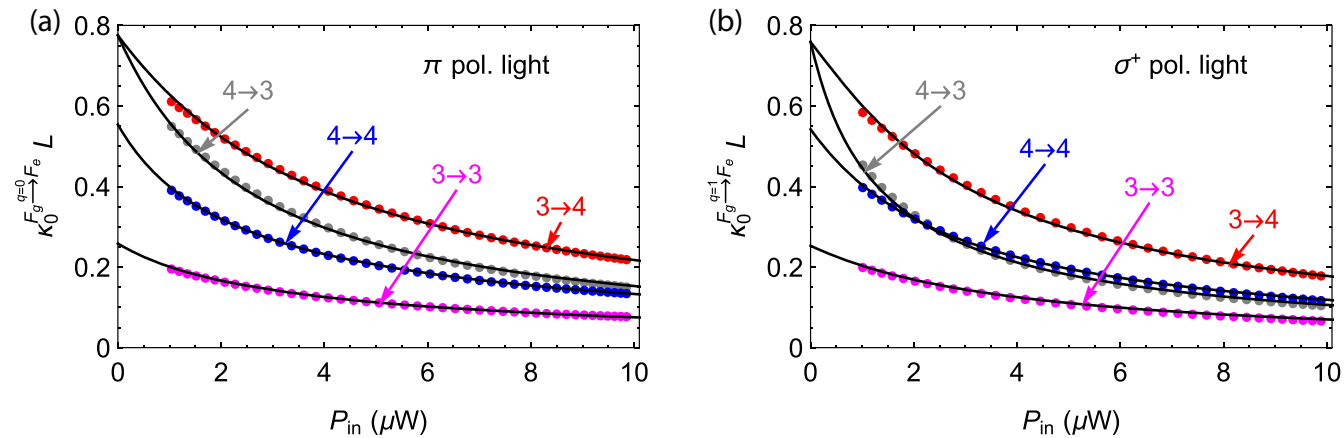

FIG. 10. Power dependence of the optical thickness $\kappa_{0}^{F_{g} \stackrel{q}{\rightarrow} F_{e}} L$ for the four hyperfine transitions measured with (a) linearly polarized and (b) circularly polarized light. The solid lines are fits using the solutions of the rate equation model including both isotropic and spin-exchange relaxation. The dots are the experimental values, where we dropped every second value (compared to Fig. 3) in order to make the fitted curves more visible.

a reasonable value for the (room) temperature at which the experiments were carried out.

\section{B. Fitted saturation power $\tilde{\boldsymbol{P}}_{\text {sat }}^{q}$}

According to Eq. (17) the theoretical expression for the saturation power is

$$
P_{\mathrm{sat}}=\frac{\hbar \Gamma_{D} \gamma_{1} S_{\mathrm{eff}}}{4 \pi^{3 / 2} \alpha|r|^{2}}
$$

We note that this expression is based on the absorption rate $\Gamma_{\text {abs }}$ derived in Appendix A 2 under the tacit assumption that the atoms interact with the laser beam sufficiently long to reach a steady state of the optical pumping process. For the relatively modest laser intensities used in our experiments, such a steady-state-reaching interaction would only be possible in a laser beam with an unrealistically large transverse extension. The high pumping efficiency obtained in an antirelaxation coated cell, like the one used here, builds on the fact that the atoms undergo multiple (spin polarization nondestructing) wall collisions so that a steady state is reached before the atom escapes into the sidearm. Because of this, each atom will reenter the laser beam multiple times and continue to be pumped on each beam traversal. Since the atoms spend most of their time in the dark volume $V_{\text {cell }}=4 \pi R_{\text {cell }}^{3} / 3$ of the cell, while being pumped only when inside of the illuminated volume $V_{\text {beam }}=2 R_{\text {cell }} S_{\text {eff }}$, we take this effect into account by replacing $\gamma_{\text {abs }}$ by an effective absorption rate

$$
\gamma_{\mathrm{abs}} \rightarrow \gamma_{\mathrm{abs}}^{\mathrm{eff}}=\frac{V_{\mathrm{beam}}}{V_{\text {cell }}} \gamma_{\mathrm{abs}}=\frac{3 S_{\mathrm{eff}}}{2 \pi R_{\mathrm{cell}}^{2}} \gamma_{\mathrm{abs}} .
$$

TABLE I. Numerical values of the three fit parameters of the data shown in Fig. 10.

\begin{tabular}{lcc}
\hline \hline & $q=0$ & \\
Parameter & $\pi$ polarization & $\sigma^{+}$polarization \\
\hline$\tilde{A}^{q}$ & $2.36(8)$ & $2.31(14)$ \\
$\tilde{P}_{\mathrm{sat}}^{q}(\mathrm{nW})$ & $58(6)$ & $43(5)$ \\
$\tilde{\varepsilon}_{\mathrm{se}}^{q}$ & $2.4(3)$ & $3.8(7)$ \\
\hline \hline
\end{tabular}

Since $x=P_{\text {in }} / P_{\text {sat }} \propto \gamma_{\text {abs }} / \gamma_{1}$, the substitution (28) is equivalent to the substitution

$$
P_{\text {sat }} \rightarrow P_{\text {sat }}^{\text {eff }}=\frac{V_{\text {cell }}}{V_{\text {beam }}} P_{\text {sat }}=\frac{\hbar \Gamma_{D} \gamma_{1} R_{\text {cell }}^{2}}{6 \pi^{1 / 2} \alpha|r|^{2}} .
$$

With $|r|^{2}, \Gamma_{D}$, and $R_{\text {cell }}=14.5(5) \mathrm{mm}$ known, we can infer from Eq. (29) the isotropic relaxation rate $\gamma_{1}$. The fitted effective saturation powers $\tilde{P}_{\text {sat }}^{q}=58(6)$ and $43(5) \mathrm{nW}$ from the experiments with linearly polarized and circularly polarized light yield $\gamma_{1} / 2 \pi=1.4(2)$ and $1.0(1) \mathrm{Hz}$, respectively. For the argument further down we will use their weighted average $\bar{\gamma}_{1} / 2 \pi=1.2(3) \mathrm{Hz}$.

In contrast to Eq. (27), Eq. (29) no longer depends on the cross section $S_{\text {eff }}$ of the laser beam. This reflects the wellknown fact among researchers working with optically pumped antirelaxation coated cells that the performance of such sensors (e.g., for magnetometry) does not have a pronounced dependence on the size of the laser beam. Nonetheless, the substitution in Eq. (29) is a crude one, since it does not take into account details of the laser beam profile or of the thermal atomic velocity distribution, effects which can, in principle, be modeled (see, e.g., Refs. [17,18]), but which are too cumbersome to implement here. The results reported above were obtained with a laser beam radius $w \approx 2 \mathrm{~mm}$. Note that we have repeated the experiments with a $w \approx 0.35 \mathrm{~mm}$ beam and have obtained compatible results.

\section{Fitted relaxation parameter $\tilde{\varepsilon}_{\mathrm{se}}^{q}$}

From the fitted $\tilde{\varepsilon}_{\mathrm{se}}^{q}\left(=\gamma_{\mathrm{se}} / \gamma_{1}\right)$ values (Table I) and the $\gamma_{1}$ values derived from $P_{\text {sat }}^{\text {eff }}$ in Sec. VB, we can estimate the spin-exchange rates $\gamma_{\mathrm{se}} / 2 \pi$ to be $3.4(6)$ and $4.0(9) \mathrm{Hz}$, with weighted average $\bar{\gamma}_{\text {se }} / 2 \pi=3.6(4) \mathrm{Hz}$. From the Cs-Cs spin-exchange cross section $\sigma_{\mathrm{se}}=2.06 \times 10^{-14} \mathrm{~cm}^{2}$ reported in Ref. [19] one can calculate the spin-exchange rate according to

$$
\gamma_{\mathrm{se}}=N_{\mathrm{Cs}}(T) \bar{v}_{\mathrm{rel}}(T) \sigma_{\mathrm{se}},
$$

where $\bar{v}_{\text {rel }}=\sqrt{\frac{16 k_{\mathrm{B}} T}{\pi M_{\mathrm{Cs}}}}$ is the average relative thermal velocity of Cs atoms. Determining the temperature $T$, for which Eq. (30) produces the measured $\bar{\gamma}_{\mathrm{se}}$ value, yields $T=22.5(1.1)^{\circ} \mathrm{C}$, a value that is compatible with the temperature derived from the signal amplitudes $\tilde{A}^{q}$ in Sec. V A. 


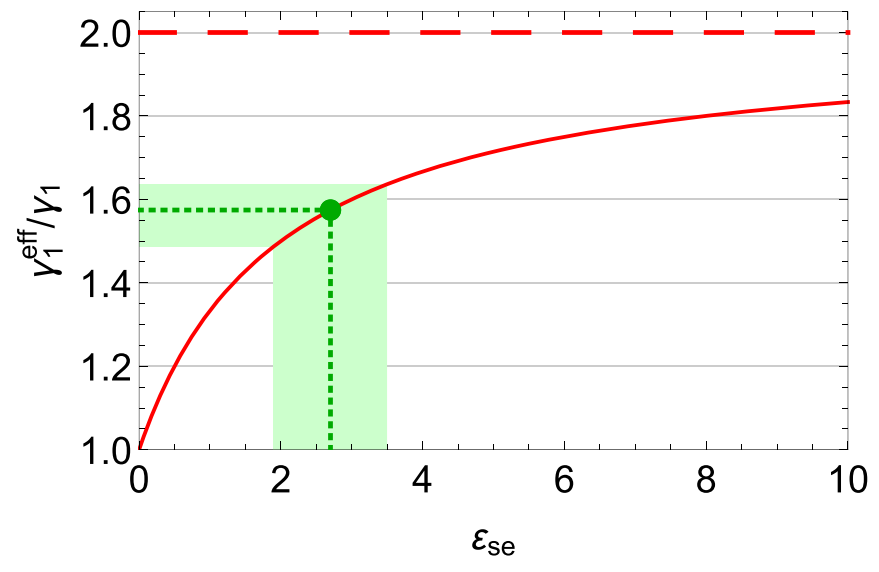

FIG. 11. Dependence of $\gamma_{1}^{\text {eff }} / \gamma_{1}$ from Eq. (31) on $\varepsilon_{\mathrm{se}}=\gamma_{\mathrm{se}} / \gamma_{1}$. The green dotted lines show how $\gamma_{1}^{\text {eff }} / \gamma_{1}$ is inferred from the fitted $\bar{\varepsilon}_{\text {se }}$ value. The dashed line marks the asymptote for $\varepsilon_{\text {se }} \rightarrow \infty$.

Equation (20) of Ref. [19], viz.,

$$
\gamma_{1}^{\text {eff }}=\gamma_{1}+\frac{1}{1 / \gamma_{1}+2 / \gamma_{\mathrm{se}}},
$$

expresses the effective longitudinal relaxation rate (in low fields) in terms of the isotropic and spin-exchange relaxation rates. Combining Eq. (31) with our definition $\varepsilon_{\mathrm{se}}=\gamma_{\mathrm{se}} / \gamma_{1}$ yields the relation

$$
\gamma_{1}^{\mathrm{eff}}=\frac{2 \varepsilon_{\mathrm{se}}+2}{\varepsilon_{\mathrm{se}}+2} \gamma_{1}
$$

between $\gamma_{1}^{\text {eff }}$ and $\gamma_{\text {se }}$.

Figure 11 shows the $\gamma_{1}^{\text {eff }} / \gamma_{1}\left(\varepsilon_{\mathrm{se}}\right)$ dependence that illustrates the bounds $\gamma_{1} \leqslant \gamma_{1}^{\text {eff }} \leqslant 2 \gamma_{1}$ of $\gamma_{1}^{\text {eff }}$. The green bands in Fig. 11 show how $\gamma_{1}^{\text {eff }} / \gamma_{1}$ can be inferred from $\bar{\varepsilon}_{\mathrm{se}}=2.7(8)$. Together with $\bar{\gamma}_{1}=1.2(3) \mathrm{Hz}$, Eq. (32) yields $\bar{\gamma}_{1}^{\text {eff }}=1.9(4) \mathrm{Hz}$, a value compatible with our measurements of similar cells using a double-resonance $M_{x}$ magnetometry method [5].

\section{SUMMARY AND OUTLOOK}

We have studied the reduction, by optical pumping with linearly and circularly polarized light, of the resonant absorption coefficients $\kappa_{0}$ of the four $6 S_{1 / 2}, F_{g} \rightarrow 6 P_{1 / 2}, F_{e}$ hyperfine components of room temperature Cs vapor contained in a paraffin-coated cell. The dependence of $\kappa_{0}$ on laser power $P_{\text {in }}$ is well described by rate equations that take both isotropic and spin-exchange relaxation of the ground-state sublevel populations into account. Fitting the model calculations with simple scaling parameters for $\kappa_{0}$ and $P_{\text {in }}$ to the experimental $\kappa_{0}\left(P_{\text {in }}\right)$ data yields consistent values of the fit parameters for all four transitions, under both linearly and circularly polarized light excitation. The scaling parameters are well described by explicit theoretical expressions on an absolute scale. The third fit parameter is the ratio $\gamma_{\text {se }} / \gamma_{1}$ of the spin-exchange and isotropic relaxation rates. When combined with an auxiliary determination of the effective longitudinal spin-relaxation rate $\gamma_{1}^{\text {eff }}\left(\gamma_{\mathrm{se}}, \gamma_{1}\right)$, one obtains absolute values of $\gamma_{\mathrm{se}}$ and $\gamma_{1}$, which are in good agreement with literature values. We have also presented algebraic expressions that describe the $\kappa_{0}\left(P_{\text {in }}\right)$ dependence in the case of isotropic relaxation only.

\section{ACKNOWLEDGMENTS}

One of us (Y.S.) acknowledges financial support from the China Scholarship Council (China), under Grant No. 201604910697 and the National Natural Science Foundation of China (China), under Grant No. 41704177.

\section{APPENDIX A: ABSORPTION COEFFICIENT AND ABSORPTION RATE}

The electric dipole matrix elements

$$
d_{b a}^{(q)}=-e x_{b a}^{(q)} \equiv-e\left\langle b\left|x_{q}\right| a\right\rangle
$$

play an important role in the definition of the absorption coefficient $\kappa_{0}$ and absorption rate $\gamma_{\text {abs }}$ discussed below. In the basis of total angular momentum states $|a\rangle=\left|F_{g}, m_{g}\right\rangle \equiv\left|6 S_{1 / 2}, F_{g}, m_{g}\right\rangle$ and $|b\rangle=\left|F_{e}, m_{e}\right\rangle \equiv$ $\left|6 P_{1 / 2}, F_{e}, m_{e}\right\rangle$, the squared matrix element between a given magnetic sublevel $\left|F_{g}, m_{g}\right\rangle$ of the ground state and the excitedstate sublevel $\left|F_{e}, m_{e}=m_{e}+q\right\rangle$ connected by absorption of light with polarization $q$ is given by

$$
\begin{aligned}
\left|x_{b a}^{(q)}\right|^{2} & =\left|x_{F_{e}, m_{e} ; F_{g}, m_{g}}^{(q)}\right|^{2} \\
& =\left|\left\langle F_{e}, m_{e}\left|x_{q}\right| F_{g}, m_{g}\right\rangle\right|^{2} \\
& \equiv c_{F_{e}, m_{e} ; F_{g}, m_{g}}^{(q)}\left|\left\langle 6 P_{1 / 2}\|r\| 6 S_{1 / 2}\right\rangle\right|^{2} \\
& \equiv c_{F_{e}, m_{e} ; F_{g}, m_{g}}^{(q)}|r|^{2},
\end{aligned}
$$

with

$$
\begin{aligned}
c_{F_{e}, m_{e} ; F_{g}, m_{g}}^{(q)}= & \left(2 F_{g}+1\right)\left(2 F_{e}+1\right) \\
& \times\left(\begin{array}{ccc}
F_{e} & 1 & F_{g} \\
-m_{e} & q & m_{g}
\end{array}\right)^{2}\left\{\begin{array}{ccc}
J_{e} & J_{g} & 1 \\
F_{g} & F_{e} & I
\end{array}\right\}^{2}
\end{aligned}
$$

and

$$
|r|=\left|\left\langle 6 P_{1 / 2}\|r\| 6 S_{1 / 2}\right\rangle\right| .
$$

In Eq. (A3), $I$ ( $=7 / 2$ for ${ }^{133} \mathrm{Cs}$ ) is the nuclear spin and the $3 j$ symbol properties imply that $m_{e}=m_{g}+q$. The decoupling rules used for the matrix elements can be found, e.g., in Ref. [20].

\section{Resonant absorption coefficient}

For an optically nonsaturating laser intensity, the resonant absorption coefficient for light of polarization $q$ (tuned to the $|a\rangle \rightarrow|b\rangle$ transition of frequency $\omega_{a b}$ ) that traverses vapor of Cs atoms with a Maxwellian velocity distribution is given by

$$
\kappa_{0}^{a} \stackrel{q}{\rightarrow} b=\frac{\sqrt{\pi} \omega_{b a}}{\varepsilon_{0} \hbar c \Gamma_{D}}\left|d_{b a}^{(q)}\right|^{2} p_{a} N_{\mathrm{Cs}},
$$

where $\varepsilon_{0}$ is the vacuum permittivity, $\hbar$ the reduced Planck constant, $c$ the vacuum light speed, $N_{\mathrm{Cs}}$ the cesium number density, $p_{a}$ the population (fraction) of atoms in state $|a\rangle$ (such that $N_{a}=p_{a} N_{\mathrm{Cs}}$ ), and

$$
\Gamma_{D}=\omega_{a b} \sqrt{\frac{2 k_{B} T}{M_{\mathrm{Cs}} c^{2}}}
$$

the Doppler width (with the Boltzmann constant $k_{B}$ and cesium atomic mass $\left.M_{\mathrm{Cs}}\right)$, using the line-shape definition $\mathcal{L}_{D}(\delta \omega)=$ 
$e^{-\delta \omega^{2} / \Gamma_{D}^{2}}$ for the Doppler profile. This result is valid in the so-called Doppler limit, in which $\Gamma_{D}$ is much larger than the natural width $\gamma_{2}^{\mathrm{opt}}$ of the transition, a condition that is well obeyed for the $D_{1}$ line of Cs at room temperature. In Eq. (A5)

$$
\left|d_{b a}^{(q)}\right|^{2}=\left|\left\langle b\left|d_{q}\right| a\right\rangle\right|^{2}=e^{2}\left|\left\langle b\left|x_{q}\right| a\right\rangle\right|^{2} \equiv e^{2}\left|x_{b a}^{(q)}\right|^{2}
$$

defines the squared modulus of the electric dipole matrix element, where $q$ describes the polarization ( $q=0$ for linearly and $q= \pm 1$ for circularly polarized light) of the absorbed light beam. Defining

$$
C_{\kappa} \equiv \frac{e^{2}}{\varepsilon_{0} \hbar} \sqrt{\frac{\pi M_{\mathrm{Cs}}}{2 k_{B} T}} N_{\mathrm{Cs}}=4 \pi \alpha \sqrt{\frac{\pi M_{\mathrm{Cs}} c^{2}}{2 k_{B} T}} N_{\mathrm{Cs}},
$$

where $\alpha$ is the fine-structure constant, Eq. (A5) reads

$$
\kappa_{0}^{a \stackrel{q}{\rightarrow} b}=p_{a}\left|x_{b a}^{(q)}\right|^{2} C_{\kappa} .
$$

Using the explicit decomposition (A2) of the matrix elements, we can rewrite the peak absorption coefficient as

$$
\kappa_{0}^{F_{g}, m_{g} \stackrel{q}{\rightarrow} F_{e}, m_{e}} \equiv p_{F_{g}, m_{g}} c_{F_{e}, m_{e} ; F_{g}, m_{g}}^{(q)} \tilde{C}_{\kappa},
$$

with

$$
\tilde{C}_{\kappa}=|r|^{2} C_{\kappa} .
$$

One can define a total absorption coefficient of the $D_{1}$ transition by

$$
\kappa_{\text {tot }} \equiv \sum_{F_{e}=I-J_{e}}^{I+J_{e}} \sum_{F_{g}=I-J_{g}}^{I+J_{g}} \sum_{m_{g}=-F_{g}}^{+F_{g}} \kappa_{0}^{F_{g}, m_{g} \stackrel{q}{\rightarrow} F_{e}, m_{e}} .
$$

Evaluating Eq. (A12) by inserting thermal populations $p_{F_{g}, m_{g}}=1 / 16$ into Eq. (A10) yields the total absorption coefficient for an unpolarized medium

$$
\begin{aligned}
\kappa_{\text {tot }}^{\text {unpol }} & =\frac{1}{16} \sum_{F_{e}=I-J_{e}}^{I+J_{e}} \sum_{F_{g}=I-J_{g}}^{I+J_{g}} \sum_{m_{g}=-F_{g}}^{+F_{g}} c_{F_{e}, m_{e} ; F_{g}, m_{g}}^{(q)} \tilde{C}_{\kappa} \\
& =\frac{1}{6} \tilde{C}_{\kappa},
\end{aligned}
$$

that is independent of $q$, as it should be, because of the isotropy of the unpolarized medium.

\section{Resonant absorption rate}

The absorption rate, i.e., the number of photons with a given polarization absorbed (on average) by an atom (at rest) in state $|a\rangle$, is given by

$$
\gamma_{\mathrm{abs}}^{a \rightarrow b}=\frac{\left|\Omega_{b a}\right|^{2}}{2 \gamma_{2}^{\mathrm{opt}}},
$$

where $\gamma_{2}^{\mathrm{opt}}$ is the transverse relaxation rate of the atomic dipole moment and

$$
\left|\Omega_{b a}\right|^{2}=\left|\frac{\vec{d}_{b a} \cdot \hat{E}}{\hbar}\right|^{2} E_{0}^{2}
$$

is the square of the optical Rabi frequency. Here $E_{0}$ and $\hat{E}$ are the amplitude and polarization vector of a plane wave, when expressed as $\vec{E}=E_{0} \hat{E} \cos (k z-\omega t)$. Introducing the light intensity $I$, given by

$$
I=\frac{\varepsilon_{0} c}{2} E_{0}^{2},
$$

the absorption rate can be expressed as

$$
\gamma_{\mathrm{abs}}^{a \rightarrow b}=\frac{1}{\varepsilon_{0} \hbar^{2} c \gamma_{2}^{\mathrm{opt}}}\left|\vec{d}_{b a} \cdot \hat{E}\right|^{2} I .
$$

We note that Eq. (A17) is valid for an atom at rest which has a Lorentzian absorption profile with a FWHM of $2 \gamma_{2}^{\text {opt }}$. The expression can be adopted to a Doppler-broadened medium, for which

$$
\kappa_{D}(\delta \omega)=\kappa_{0}^{D} e^{-\delta \omega^{2} / \Gamma_{D}^{2}}=\frac{\sqrt{\pi} \gamma_{2}^{\mathrm{opt}}}{\Gamma_{D}} \kappa_{0} e^{-\delta \omega^{2} / \Gamma_{D}^{2}},
$$

by replacing $\gamma_{2}^{\text {opt }}$ in Eq. (A17) by $\Gamma_{D} / \sqrt{\pi}$, yielding

$$
\gamma_{\mathrm{abs}}^{a \rightarrow b}=\frac{\sqrt{\pi}\left|\vec{d}_{b a} \cdot \hat{E}\right|^{2}}{\varepsilon_{0} \hbar^{2} c \Gamma_{D}} I .
$$

Introducing spherical tensor notation

$$
d^{( \pm 1)}=\mp \frac{1}{\sqrt{2}}\left(d^{(x)} \pm i d^{(y)}\right), \quad d^{(0)}=d^{(z)}
$$

for the components $d^{(q=0, \pm 1)}$ of the dipole operator and similarly for the components $E_{q}$ of the polarization vector, the scalar product in Eq. (A17), which reads

$$
\vec{d}_{b a} \cdot \hat{E}=\sum d_{b a}^{(i)} E_{i}=d^{(x)} E_{x}+d^{(y)} E_{y}+d^{(z)} E_{z}
$$

in Cartesian coordinates, will read

$$
\begin{aligned}
\vec{d} \cdot \hat{E} & =\sum_{q=0, \pm 1}(-1)^{q} d_{b a}^{(q)} E_{-q} \\
& =d^{(0)} E_{0}-d^{(+1)} E_{-1}-d^{(-1)} E_{+1}
\end{aligned}
$$

in spherical coordinates. The components $E_{0}$ and $E_{ \pm 1}$ represent $\pi$ - (linearly) and $\sigma^{ \pm 1}$ - (circularly) polarized light, respectively. For light described by the (unit) polarization vector $E_{-q}$, only the matrix elements of the dipole operator component $d^{(q)}$ will be responsible for the light absorption, so Eq. (A17) reduces to

$$
\gamma_{\mathrm{abs}}^{a \stackrel{q}{\rightarrow} b}=\frac{\sqrt{\pi} e^{2}}{\varepsilon_{0} \hbar^{2} c \Gamma_{D}}\left|x_{b a}^{(q)}\right|^{2} I,
$$

where we have used $d_{b a}^{(q)}=e x_{b a}^{(q)}$.

Similarly to the parametrization used for the absorption coefficient in Appendix A 1, we can parametrize the absorption rate on the $|a\rangle \rightarrow|b\rangle$ transition driven by $q$-polarized light as

$$
\gamma_{\mathrm{abs}}^{a \stackrel{q}{\rightarrow} b}=C_{\gamma_{\mathrm{abs}}}\left|x_{b a}^{(q)}\right|^{2}
$$

with

$$
C_{\gamma_{\mathrm{abs}}}=\frac{\sqrt{\pi} e^{2}}{\varepsilon_{0} \hbar^{2} c \Gamma_{D}} I=\frac{4 \pi^{3 / 2} \alpha}{\hbar \Gamma_{D}} \frac{P_{\mathrm{in}}}{S_{\mathrm{eff}}},
$$

where $\alpha$ is the fine-structure constant. We also have expressed the light intensity $I \equiv P_{\text {in }} / S_{\text {eff }}$ as power per unit area, introducing an effective cross-section area $S_{\text {eff }}$ characterizing 
light beams with a nonhomogeneous transverse intensity distribution. Using the explicit decomposition (A2) of the matrix elements, we can rewrite the absorption rate of Eq. (A25) as

$$
\begin{aligned}
\gamma_{\mathrm{abs}}^{F_{g}, m_{g} \stackrel{q}{\rightarrow} F_{e}, m_{e}} & =c_{F_{e}, m_{e} ; F_{g}, m_{g}}^{(q)}|r|^{2} C_{\gamma_{\mathrm{abs}}} \\
& \equiv c_{F_{e}, m_{e} ; F_{g}, m_{g}}^{(q)} \Gamma_{\mathrm{abs}},
\end{aligned}
$$

with

$$
\Gamma_{\mathrm{abs}}=\frac{4 \pi^{3 / 2} \alpha|r|^{2}}{\hbar \Gamma_{\mathrm{D}}} \frac{P_{\mathrm{in}}}{S_{\mathrm{eff}}}
$$

We recall that $\gamma_{\mathrm{abs}}^{F_{g}, m_{g} \stackrel{q}{\rightarrow} F_{e}, m_{e}}$ is a property of individual atoms that is proportional to laser power, while $\kappa_{0}^{F_{g} \stackrel{q}{\rightarrow} F_{e}}$ is an ensemble property that is proportional to the density $N_{\mathrm{Cs}}$ of atoms in the $6 S_{1 / 2}$ ground state.

\section{APPENDIX B: MATRIX REPRESENTATION OF THE SPIN OPERATORS $\mathbb{S}_{x, y, z}$}

The spherical components $\mathbb{S}_{q=-1,0,+1}$ of the total angular momentum operator are defined in terms of the Cartesian components $\mathbb{S}_{i=x, y, z}$ by

$$
\mathbb{S}_{ \pm 1}=\mp \frac{\mathbb{S}_{x} \pm i \mathbb{S}_{y}}{\sqrt{2}}, \quad \mathbb{S}_{0}=\mathbb{S}_{z}
$$

so the inverse relations read

$$
\mathbb{S}_{x}=\frac{\mathbb{S}_{-1}-\mathbb{S}_{+1}}{\sqrt{2}}, \quad \mathbb{S}_{y}=i \frac{\mathbb{S}_{-1}+\mathbb{S}_{+1}}{\sqrt{2}}, \quad \mathbb{S}_{z}=\mathbb{S}_{0} .
$$

In the basis of total angular momentum states $|F, m\rangle \equiv$ $|n, I, S ; F, m\rangle$, the matrix elements of the operators $\mathbb{S}_{q}$ are given by

$$
\left\langle F^{\prime}, m^{\prime}|\mathbb{S}| F, m\right\rangle=(-1)^{F^{\prime}-m^{\prime}}\left(\begin{array}{ccc}
F^{\prime} & 1 & F \\
-m^{\prime} & q & m
\end{array}\right)\left\langle n, I, S ; F^{\prime}\|\mathbb{S}\| n, I, S ; F\right\rangle
$$

where we have used the Wigner-Eckart theorem. The reduced matrix element is given by

$$
\begin{aligned}
& \left\langle n, I, S ; F^{\prime}\|\mathbb{S}\| n, I, S ; F\right\rangle=(-1)^{S+1+I+F^{\prime}} \sqrt{2 F+1} \sqrt{2 F^{\prime}+1}\left\{\begin{array}{ccc}
S & F^{\prime} & I \\
F & S & 1
\end{array}\right\}\langle S\|\mathbb{S}\| S\rangle \text {. } \\
& =(-1)^{F^{\prime}+1} \sqrt{\frac{3(2 F+1)\left(2 F^{\prime}+1\right)}{2}}\left\{\begin{array}{ccc}
1 / 2 & F^{\prime} & 7 / 2 \\
F & 1 / 2 & 1
\end{array}\right\} \text {. }
\end{aligned}
$$

using relations from Ref. [20]. Combining Eqs. (B2)-(B4), one obtains the following matrix representations of the operators $\mathbb{F}_{x, y, z}$ in the basis $\left\{\left|F_{g}, m_{g}\right\rangle\right\}=\{|3,-3\rangle, \ldots,|3,+3\rangle,|4,-4\rangle, \ldots,|4,+4\rangle\}$ :

$$
\mathbb{S}_{x}=\frac{1}{8 \sqrt{2}}\left(\begin{array}{cccccccccccccccc}
0 & -\sqrt{3} & 0 & 0 & 0 & 0 & 0 & -2 \sqrt{7} & 0 & 1 & 0 & 0 & 0 & 0 & 0 & 0 \\
-\sqrt{3} & 0 & -\sqrt{5} & 0 & 0 & 0 & 0 & 0 & -\sqrt{21} & 0 & \sqrt{3} & 0 & 0 & 0 & 0 & 0 \\
0 & -\sqrt{5} & 0 & -\sqrt{6} & 0 & 0 & 0 & 0 & 0 & -\sqrt{15} & 0 & \sqrt{6} & 0 & 0 & 0 & 0 \\
0 & 0 & -\sqrt{6} & 0 & -\sqrt{6} & 0 & 0 & 0 & 0 & 0 & -\sqrt{10} & 0 & \sqrt{10} & 0 & 0 & 0 \\
0 & 0 & 0 & -\sqrt{6} & 0 & -\sqrt{5} & 0 & 0 & 0 & 0 & 0 & -\sqrt{6} & 0 & \sqrt{15} & 0 & 0 \\
0 & 0 & 0 & 0 & -\sqrt{5} & 0 & -\sqrt{3} & 0 & 0 & 0 & 0 & 0 & -\sqrt{3} & 0 & \sqrt{21} & 0 \\
0 & 0 & 0 & 0 & 0 & -\sqrt{3} & 0 & 0 & 0 & 0 & 0 & 0 & 0 & -1 & 0 & 2 \sqrt{7} \\
-\sqrt{7} & 0 & 0 & 0 & 0 & 0 & 0 & 0 & 2 & 0 & 0 & 0 & 0 & 0 & 0 & 0 \\
0 & -\sqrt{21} & 0 & 0 & 0 & 0 & 0 & 2 & 0 & \sqrt{7} & 0 & 0 & 0 & 0 & 0 & 0 \\
1 & 0 & -\sqrt{15} & 0 & 0 & 0 & 0 & 0 & \sqrt{7} & 0 & 3 & 0 & 0 & 0 & 0 & 0 \\
0 & \sqrt{3} & 0 & -\sqrt{10} & 0 & 0 & 0 & 0 & 0 & 3 & 0 & \sqrt{10} & 0 & 0 & 0 & 0 \\
0 & 0 & \sqrt{6} & 0 & -\sqrt{6} & 0 & 0 & 0 & 0 & 0 & \sqrt{10} & 0 & \sqrt{10} & 0 & 0 & 0 \\
0 & 0 & 0 & \sqrt{10} & 0 & -\sqrt{3} & 0 & 0 & 0 & 0 & 0 & \sqrt{10} & 0 & 3 & 0 & 0 \\
0 & 0 & 0 & 0 & \sqrt{15} & 0 & -1 & 0 & 0 & 0 & 0 & 0 & 3 & 0 & \sqrt{7} & 0 \\
0 & 0 & 0 & 0 & 0 & \sqrt{21} & 0 & 0 & 0 & 0 & 0 & 0 & 0 & \sqrt{7} & 0 & 2 \\
0 & 0 & 0 & 0 & 0 & 0 & 2 \sqrt{7} & 0 & 0 & 0 & 0 & 0 & 0 & 0 & 2 & 0
\end{array}\right)
$$




$$
\mathbb{S}_{y}=\frac{i}{8 \sqrt{2}}\left(\begin{array}{cccccccccccccccc}
0 & -\sqrt{3} & 0 & 0 & 0 & 0 & 0 & 2 \sqrt{7} & 0 & 1 & 0 & 0 & 0 & 0 & 0 & 0 \\
\sqrt{3} & 0 & -\sqrt{5} & 0 & 0 & 0 & 0 & 0 & \sqrt{21} & 0 & \sqrt{3} & 0 & 0 & 0 & 0 & 0 \\
0 & \sqrt{5} & 0 & -\sqrt{6} & 0 & 0 & 0 & 0 & 0 & \sqrt{15} & 0 & \sqrt{6} & 0 & 0 & 0 & 0 \\
0 & 0 & \sqrt{6} & 0 & -\sqrt{6} & 0 & 0 & 0 & 0 & 0 & \sqrt{10} & 0 & \sqrt{10} & 0 & 0 & 0 \\
0 & 0 & 0 & \sqrt{6} & 0 & -\sqrt{5} & 0 & 0 & 0 & 0 & 0 & \sqrt{6} & 0 & \sqrt{15} & 0 & 0 \\
0 & 0 & 0 & 0 & \sqrt{5} & 0 & -\sqrt{3} & 0 & 0 & 0 & 0 & 0 & \sqrt{3} & 0 & \sqrt{21} & 0 \\
0 & 0 & 0 & 0 & 0 & \sqrt{3} & 0 & 0 & 0 & 0 & 0 & 0 & 0 & 1 & 0 & 2 \sqrt{7} \\
-2 \sqrt{7} & 0 & 0 & 0 & 0 & 0 & 0 & 0 & 2 & 0 & 0 & 0 & 0 & 0 & 0 & 0 \\
0 & -\sqrt{21} & 0 & 0 & 0 & 0 & 0 & -2 & 0 & \sqrt{7} & 0 & 0 & 0 & 0 & 0 & 0 \\
-1 & 0 & -\sqrt{15} & 0 & 0 & 0 & 0 & 0 & -\sqrt{7} & 0 & 3 & 0 & 0 & 0 & 0 & 0 \\
0 & -\sqrt{3} & 0 & -\sqrt{10} & 0 & 0 & 0 & 0 & 0 & -3 & 0 & \sqrt{10} & 0 & 0 & 0 & 0 \\
0 & 0 & -\sqrt{6} & 0 & -\sqrt{6} & 0 & 0 & 0 & 0 & 0 & -\sqrt{10} & 0 & \sqrt{10} & 0 & 0 & 0 \\
0 & 0 & 0 & -\sqrt{10} & 0 & -\sqrt{3} & 0 & 0 & 0 & 0 & 0 & -\sqrt{10} & 0 & 3 & 0 & 0 \\
0 & 0 & 0 & 0 & -\sqrt{15} & 0 & -1 & 0 & 0 & 0 & 0 & 0 & -3 & 0 & \sqrt{7} & 0 \\
0 & 0 & 0 & 0 & 0 & -\sqrt{21} & 0 & 0 & 0 & 0 & 0 & 0 & 0 & -\sqrt{7} & 0 & 2 \\
0 & 0 & 0 & 0 & 0 & 0 & -2 \sqrt{7} & 0 & 0 & 0 & 0 & 0 & 0 & 0 & -2 & 0
\end{array}\right)
$$

(B6)

$$
\mathbb{S}_{z}=\frac{1}{8}\left(\begin{array}{cccccccccccccccc}
3 & 0 & 0 & 0 & 0 & 0 & 0 & 0 & -\sqrt{7} & 0 & 0 & 0 & 0 & 0 & 0 & 0 \\
0 & 2 & 0 & 0 & 0 & 0 & 0 & 0 & 0 & -2 \sqrt{3} & 0 & 0 & 0 & 0 & 0 & 0 \\
0 & 0 & 1 & 0 & 0 & 0 & 0 & 0 & 0 & 0 & -\sqrt{15} & 0 & 0 & 0 & 0 & 0 \\
0 & 0 & 0 & 0 & 0 & 0 & 0 & 0 & 0 & 0 & 0 & -4 & 0 & 0 & 0 & 0 \\
0 & 0 & 0 & 0 & -1 & 0 & 0 & 0 & 0 & 0 & 0 & 0 & -\sqrt{15} & 0 & 0 & 0 \\
0 & 0 & 0 & 0 & 0 & -2 & 0 & 0 & 0 & 0 & 0 & 0 & 0 & -2 \sqrt{3} & 0 & 0 \\
0 & 0 & 0 & 0 & 0 & 0 & -3 & 0 & 0 & 0 & 0 & 0 & 0 & 0 & -\sqrt{7} & 0 \\
0 & 0 & 0 & 0 & 0 & 0 & 0 & -4 & 0 & 0 & 0 & 0 & 0 & 0 & 0 & 0 \\
-\sqrt{7} & 0 & 0 & 0 & 0 & 0 & 0 & 0 & -3 & 0 & 0 & 0 & 0 & 0 & 0 & 0 \\
0 & -2 \sqrt{3} & 0 & 0 & 0 & 0 & 0 & 0 & 0 & -2 & 0 & 0 & 0 & 0 & 0 & 0 \\
0 & 0 & -\sqrt{15} & 0 & 0 & 0 & 0 & 0 & 0 & 0 & -1 & 0 & 0 & 0 & 0 & 0 \\
0 & 0 & 0 & -4 & 0 & 0 & 0 & 0 & 0 & 0 & 0 & 0 & 0 & 0 & 0 & 0 \\
0 & 0 & 0 & 0 & -\sqrt{15} & 0 & 0 & 0 & 0 & 0 & 0 & 0 & 1 & 0 & 0 & 0 \\
0 & 0 & 0 & 0 & 0 & -2 \sqrt{3} & 0 & 0 & 0 & 0 & 0 & 0 & 0 & 2 & 0 & 0 \\
0 & 0 & 0 & 0 & 0 & 0 & -\sqrt{7} & 0 & 0 & 0 & 0 & 0 & 0 & 0 & 3 & 0 \\
0 & 0 & 0 & 0 & 0 & 0 & 0 & 0 & 0 & 0 & 0 & 0 & 0 & 0 & 0 & 4
\end{array}\right) .
$$

We note that the explicit expressions above for the matrix representations of $\mathbb{S}_{x, y, z}$ are identical to the corresponding matrices $\Sigma_{x, y, z} / 2$ (disregarding a few typographical errors) given by Scholtes et al. [14], considering the alternative ordering of the basis states used in Ref. [14].

[1] A. Kastler, Science 158, 214 (1967).

[2] D. Budker and D. F. J. Kimball, Optical Magnetometry (Cambridge University Press, London, 2013).

[3] I. Kominis, T. Kornack, J. Allred, and M. Romalis, Nature (London) 422, 596 (2003).
[4] Z. Grujić, J. Piller, and A. Weis (to be published).

[5] N. Castagna, G. Bison, G. Di Domenico, A. Hofer, P. Knowles, C. Macchione, H. Saudan, and A. Weis, Appl. Phys. B 96, 763 (2009).

[6] E. Breschi and A. Weis, Phys. Rev. A 86, 053427 (2012). 
[7] E. Breschi, Z. Grujić, and A. Weis, Appl. Phys. B 115, 85 (2014).

[8] N. Castagna and A. Weis, Phys. Rev. A 84, 053421 (2011).

[9] Wolfram Mathematica 10.4, http://www.wolfram.com/ mathematica/

[10] A. P. Kazantsev, V. S. Smirnov, A. M. Turmaikin, and I. A. Yagofarov, Opt. Spektrosk. 57, 116 (1984).

[11] F. Grossetête, J. Phys. (Paris) 25, 383 (1964).

[12] F. Grossetête, J. Phys. (Paris) 29, 456 (1968).

[13] W. Happer and W. Van Wijngaarden, Hyperfine Interact. 38, 435 (1987).

[14] T. Scholtes, S. Pustelny, S. Fritzsche, V. Schultze, R. Stolz, and H.-G. Meyer, Phys. Rev. A 94, 013403 (2016).
[15] R. J. Rafac, C. E. Tanner, A. E. Livingston, K. W. Kukla, H. G. Berry, and C. A. Kurtz, Phys. Rev. A 50, R1976 (1994).

[16] M. Rozwadowski and E. Lipworth, J. Chem. Phys. 43, 2347 (1965).

[17] E. Pfleghaar, J. Wurster, S. Kanorsky, and A. Weis, Opt. Commun. 99, 303 (1993).

[18] S. I. Kanorsky, A. Weis, and J. Skalla, Appl. Phys. B 60, S165 (1995).

[19] N. W. Ressler, R. H. Sands, and T. E. Stark, Phys. Rev. 184, 102 (1969).

[20] I. I. Sobelman, Atomic Spectra and Radiative Transitions (Springer, Berlin, 1992). 